\title{
Nanoscale heat transport at plasmonic interfaces and in 2D crystals
}

\author{
Simone Pisana \\ Lassonde School of Engineering \\ York University, Canada
}

Heat transport critically affects device performance and reliability. At the nanoscale, analysis of heat transport is complicated by finite size effects, interactions between different energy carriers and onset of ballistic phenomena. In this talk I will overview two examples of nanoscale heat transport problems that have been studied in our group. First, I will discuss energy transfer mechanisms at metal/dielectric interfaces that are critical in applications such as plasmonic devices. I will show how the use of $\sim 2 \mathrm{~nm}$ metallic layers can dramatically alter interface heat transport and outline the major factors that control it, including electron-phonon coupling and vibrational properties of the materials involved. Then, I will focus on heat transport in 2D crystals of interest for novel device applications. I will outline how the highly anisotropic transport can be measured and dramatic nanoscale transport perturbations that take place in graphene, hBN and MoS2 aggregates or WS2 single crystals. 